\title{
Study on the downward movement of carbofuran in two Malaysian soils
}

\begin{abstract}
The downward movement of carbofuran in two Malaysian soil types was studied using soil columns. The columns were filled with disturbed and undisturbed soils of either the Bagan Datoh soil (clay) or the Labu soil (sandy clay). The average total percentage of carbofuran in the leachate of the undisturbed Labu soil after 14 days of watering (80.8\%) was approximately similar to that of the total amount from the disturbed soil $(81.4 \%)$. However, carbofuran leaching was observed in the disturbed soil after the fourth day of watering whereas for the undisturbed soil, leaching occurred after the first watering. A similar trend was observed in the Bagan Datoh soil where the residue of carbofuran was detected after the first day of watering in the undisturbed soil column but only at the eighth day of watering in the disturbed soil column. The total percentage carbofuran in the leachate of disturbed and undisturbed soil columns from Bagan Datoh after 14 days of watering was $3.6 \%$ and $41.7 \%$, respectively. The study showed that less leaching occurred in soil columns with high organic content such as the Bagan Datoh soil and especially so in disturbed soils where the organic matter was homogeneously mixed in all layers.
\end{abstract}

Keyword: Carbofuran; Leaching; Clay; Sandy clay; Soil 